\title{
The role of overt language production in the Hebb repetition effect
}

\author{
Marie-Claude Guerrette $^{1,2} \cdot$ Katherine Guérard $^{1}$ - Jean Saint-Aubin ${ }^{1}$
}

Published online: 13 February 2017

(C) Psychonomic Society, Inc. 2017

\begin{abstract}
The Hebb repetition effect (Hebb, 1961) occurs when recall performance improves for a list that is repeated during a serial-recall task. This effect is considered a good experimental analogue to language learning. Our objective was to evaluate the role of overt language production in language learning by manipulating recall direction during a Hebb repetition paradigm. In each trial, seven nonsense syllables were presented auditorily. Participants had to orally recall the items either in the presentation order or in reverse order. One sequence was repeated every third trial. In Experiment 1, we compared learning from a group that had recalled the items in their presentation order to learning from a group that had recalled the items in the reverse order. The two groups yielded similar learning rates. In Experiment 2, recall direction was varied between trials. The learning rate was not affected when recall direction varied between trials, suggesting a limited role of overt language production in language learning.
\end{abstract}

Keywords Hebb repetition effect - Backward recall · Overt language production $\cdot$ Sequence learning

The processes that underpin sequence learning have fascinated researchers for decades. To study sequence learning, many researchers have used the Hebb repetition paradigm. In such a task, participants are presented with lists of digits, letters, or words that they must recall in their presentation order, and one

Marie-Claude Guerrette

marie-claude.guerrette@umontreal.ca

1 École de psychologie, Université de Moncton, Moncton E1A 3E9, New Brunswick, Canada

2 Département de psychologie, Université de Montréal, Montréal, Québec H2V 2S9, Canada list is repeated at a regular interval (see Hebb, 1961). Hebb observed that recall performance for the repeated list increased substantially over repetitions, as compared to recall for nonrepeated lists. This effect is known as the Hebb repetition effect. The Hebb repetition effect is commonly used as a laboratory analogue to language learning (Hitch, Flude, \& Burgess, 2009; Mosse \& Jarrold, 2008; Page, Cumming, Norris, Hitch, \& McNeil, 2006; Page, Cumming, Norris, McNeil, \& Hitch, 2013; Page \& Norris, 2009; Szmalec, Duyck, Vandierendonck, Mata, \& Page, 2009). Indeed, Hebbian learning and language learning both involve the perception of repeatedly presented lists of verbal items, followed by subsequent reproduction of the items in the correct order. However, one question that has received limited attention is the role of language production in language learning. Existing models of memory (see Burgess \& Hitch, 2006; Page \& Norris, 2009) suggest that the perception of the list is the main process underlying learning in the Hebb repetition effect. However, some evidence has suggested a role of language production in the Hebb effect (Cohen \& Johansson, 1967; Cunningham, Healy, \& Williams, 1984). In the present article, we therefore examined the role of overt language production in the Hebb repetition effect by manipulating the order in which the lists of items to retain had to be output during oral recall.

One model that has been extended to account for the Hebb repetition effect is the primacy model, proposed by Page and Norris (2009). The primacy model suggests that four layers of representations exist. The first layer is the occurrence layer, where units fire to signal the occurrence of their corresponding items in the world. The activation in the occurrence layer is forwarded to the recognition layer, where units compete for activation. When one unit wins the competition, the item is recognized, and activation is forwarded to the order layer, where items are associated with a primacy gradient of activation. The gradient represents a level of activation that decays exponentially for each 
successive unit. The units and their primacy gradient are then copied back to the occurrence layer. During recall, the production layer selects the most activated unit from the order layer, followed by its inhibition in order to prevent its repeated output. The production layer also changes the outgoing weights of the selected unit, which creates a steeper primacy gradient.

According to the primacy model, the Hebb repetition effect occurs in the occurrence layer, to which the primacy gradient is copied back from the order layer. When a nonrepeated list is presented, an uncommitted chunk - that is, a unit that has not seen any learning - is selected. When a repeated list is presented, however, the engaged chunk activated by the prior presentation of the list is likely to be selected. Every subsequent presentation of the same list therefore allows the engaged chunk to gain additional activation from the order layer and to be better recalled. After several presentations, the chunk becomes completely learned and is considered committed to the list. Once a chunk is learned, it is only activated when the correct items are presented in the correct order. In sum, learning is the result of repeated presentations allowing new information to be committed to chunks.

Page and Norris's (2009) model suggests that the perception of the repeated list is the main process underlying long-term acquisition of serial-order information (see also Burgess \& Hitch, 2006). In line with this idea, some studies showed that changes to the perception of the sequences disrupt the Hebb repetition effect and that language production played little role (Schwartz \& Bryden 1971). For instance, Bower and Winzenz (1969; see also Hitch et al., 2009) showed that the Hebb repetition effect was impaired when the timing to induce grouping was changed across repetitions of the repeated list. O'Shea and Clegg (2006) also investigated the effect of changing the grouping pattern only during recall and found that this manipulation had little or no consequence on the rate of learning. Similarly, Gathercole, Service, Hitch, Adams, and Martin (1999) found that children's speech output during an immediate serial-recall task was unnecessary for sequence learning. Other studies showed that preventing articulation using articulatory suppression interfered with immediate serial recall, but not with the learning of repeated lists (Hitch et al., 2009; Page et al., 2006), suggesting that speech production is unnecessary to produce a Hebb repetition effect.

Although some evidence suggests that language production is not critical in the Hebb repetition effect, other studies have suggested that language production is essential for sequence learning (Cohen \& Johansson, 1967; Cunningham et al., 1984; Mechanic, 1964; Sanders, 1961). For instance, Cohen and Johansson proposed that recall was mandatory for the emergence of a Hebb effect. They presented participants with lists of digits, with a repeated list every third trial. Immediately after each list presentation, they cued whether the list was to be recalled. The authors found that when the repeated list was never cued for recall, learning was no better than that for the nonrepeated lists, therefore suggesting that response production during recall plays an important role in the Hebb repetition effect (see also Oberauer \& Meyer, 2009).

The debate about the role of language production in the Hebb repetition effect may not generalize well to verbal learning. In effect, whereas verbal learning implies the formation of associations between an ordered set of phonemes and a higherlevel representation that has no a-priori relation, typical experiments on the Hebb repetition effect involved stimuli with well-established long-term associations. For example, most experiments using the Hebb repetition task used digits (Cumming, Page, \& Norris, 2003; Oberauer \& Meyer, 2009), letters (Cunningham et al., 1984; Kalm \& Norris, 2016), words (Page et al., 2006; Page et al., 2013), or pictures of well-known objects (Mosse \& Jarrold, 2008) as stimuli. Moreover, although language learning implies the auditory perception and a verbal production of words, most studies departed from the natural context of language learning by using visually presented sequences (Saint-Aubin, Guérard, Fiset, \& Losier, 2015; Szmalec et al., 2009) or written recall (Hitch et al., 2009; Oberauer \& Meyer, 2009; Page et al., 2013). Finally, previous studies with verbal materials had minimized the overlap between the repeated list and the nonrepeated lists (Hitch et al., 2009; Page et al., 2013), whereas words from all languages are made from a limited set of phonemes. In the present study, we used a Hebb paradigm that was as close as possible to the natural language-learning experience: Stimuli were presented auditorily and had to be recalled orally. We used a pool of 36 nonsense syllables as the stimuli to mimic the pool of 36 phonemes in the French language. The syllables in each list were selected randomly so that the same syllables could appear in repeated and nonrepeated lists.

To study the role of overt language production in the Hebb repetition effect, we manipulated recall direction using forward and backward serial recall. Backward serial recall is a variant of the typical serial-recall task in which participants are asked to recall the items in reverse order, from the last presented item to the first presented item. Backward recall allowed us to manipulate overt production order instead of production opportunity (see Cohen \& Johansson, 1967; Kalm \& Norris, 2016). Indeed, in previous experiments involving trials without a recall requirement, the mechanisms involved in those trials were unclear; retrieval of the items and the production process might already have been activated by the time the participants were instructed not to recall the list. Moreover, trials without a recall requirement do not produce any empirical data, which prevents examining the learning rate during the task. Requiring recall at every trial allows overt production processes to remain rigorously controlled throughout the experiment.

According to the primacy model (Page \& Norris, 1998), backward serial recall consists of a series of covert forward recalls. More precisely, during a backward recall, the first one or two response items could be recalled without any forward scans, since those items were presented most recently 
(see also Anders \& Lillyquist, 1971; St Clair-Thompson \& Allen, 2013). This would translate into short response times for the first one or two output items. Then, participants would resort to covert forward recalls of the list minus the last one or two items already output, followed by a reversal and output of the last few items of the list. Covert forward recalls and the output of groups of items are repeated until all items from the list are recalled in reverse order. Overall, this translates into slower response times for some middle response items, since forward covert scans take time before groups of items can be output. Output of the final response items should be relatively quicker, since the forward covert scan would have been done prior to output of the second-to-last item.

In line with Page and Norris's (1998) idea, Thomas, Milner, and Haberlandt (2003) reported a flat response time curve following the output of the first item in forward recall, but rather decreasing response times for backward recall. They argued that during forward recall, output times are constant because participants only need to retrieve each item once, from first to last. In backward recall, output times decrease with each response, because the list to retrieve becomes one item shorter after every output. Although Bireta et al. (2010) used a reconstruction task, they failed to replicate Thomas et al.'s (2003) results, therefore casting doubt on the hypothesis that backward recall implies a forward covert scan before the output of every single item. In the present experiments, we measured response times in order to assess whether participants were making a series of forward recalls during backward recall. This control is important, because in the present study we assumed that in backward recall the presentation and overt production orders would be different.

In Experiment 1, we tested the presence of a Hebb repetition effect in backward serial recall. We compared learning in a group for which all sequences were to be recalled in the forward order (forward-recall group) to a group in which all lists were to be recalled in the backward order (backward-recall group). In Experiment 2, we manipulated the recall direction of the repeated list across trials, so that the repeated sequence sometimes had to be recalled in forward order and sometimes in backward order. If overt language production plays a role in learning, changing the overt production order of the repeated list across trials should impair the Hebb repetition effect.

\section{Experiment 1}

Experiment 1 included two groups of participants. The participants in the forward-recall group always had to recall the items in their presentation order, from the first to the last nonsense syllable. The participants in the backward-recall group always had to recall the items in reverse order, from the last to the first nonsense syllable. We compared recall performance between these groups in order to evaluate the impact of backward recall on the Hebb repetition effect.

\section{Method}

Participants The forward-recall group included 20 participants ( 15 women, five men; mean age $=18.7, S D=1.3$ ); the backward-recall group also included 20 participants ( 13 women, seven men; mean age $=18.8, S D=1.2$ ). All participants were French-speaking students from Université de Moncton, and all took part in the experiment in exchange for class credits. All participants were naive to the purpose of the experiment.

Apparatus and materials The experiment was controlled by a PC computer using E-Prime (Psychology Software Tool, Inc., Version 2.0). We created 36 nonsense syllables from the combination of 16 consonants and 11 phonetic vowels. All syllables consisted of one onset consonant phoneme followed by one offset vowel phoneme; all syllables used in the experiment are presented in the Appendix. The syllables were digitally recorded with VRS Recording System (NCH Software, Version 5.48), and edited with Sound Forge Audio Studio system (Sony Corporation, Version 10.0). No syllable was to sound like a genuine French word.

\begin{tabular}{|c|c|}
\hline be & $/ \mathrm{b} /$ \\
\hline bun & 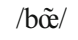 \\
\hline chin & $\mid \tilde{\varepsilon}$ \\
\hline chon & $1 \tilde{T}$ \\
\hline chu & $/ \mathrm{y} /$ \\
\hline $\mathrm{da}$ & $/ \mathrm{da} /$ \\
\hline dun & 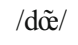 \\
\hline faï & $/ \mathrm{fj} /$ \\
\hline fun & /fீ̃̄/ \\
\hline gaï & $/ \mathrm{j} /$ \\
\hline $\mathrm{gu}$ & $/ \mathrm{y} /$ \\
\hline ja & $/ \mathrm{a} /$ \\
\hline jin & $\mid \tilde{\varepsilon}$ \\
\hline jo & $/ \mathrm{o} /$ \\
\hline kin & $/ \mathrm{k} \varepsilon \tilde{l}$ \\
\hline ko & /ko/ \\
\hline lo & $/ \mathrm{lo} /$ \\
\hline lun & /lø̃e/ \\
\hline meu & $/ \mathrm{m} \varnothing /$ \\
\hline mun & /mõe/ \\
\hline na & /na/ \\
\hline nan & /nã/ \\
\hline paï & $/ \mathrm{pj} /$ \\
\hline pé & $/ \mathrm{pe} /$ \\
\hline reu & $/ \varnothing /$ \\
\hline sun & /sõe/ \\
\hline teu & $/ \mathrm{t} \varnothing /$ \\
\hline $\mathrm{ti}$ & $/ \mathrm{ti} /$ \\
\hline vaï & $/ \mathrm{vj} /$ \\
\hline
\end{tabular}


vé

za

zan

zé

zi

zon

$\mathrm{zu}$

For each participant, at the beginning of the experiment, the program generated one repeated list and 34 nonrepeated lists. Each list was composed of seven nonsense syllables randomly drawn without replacement from the pool of 36 syllables. The entire pool of 36 nonsense syllables was used to generate each list. Consequently, no syllable was repeated within a list, but they repeated across lists. The experiment included 50 trials. The repeated list was first presented on the third trial, and then every third subsequent trial, for a total of 16 repetitions. The last two trials of the experiment were nonrepeated lists. The lists were presented auditorily through loudspeakers. A digital voice recorder was used to record the participants' answers.

Procedure All participants were tested individually in a quiet room. The experiment started with a familiarization phase during which the 36 nonsense syllables were presented visually on the computer screen and auditorily in a simultaneous fashion, at a rate of one item per 1,500 ms. The main purpose of this phase was to familiarize participants with the nonsense syllables prior to the experimental trials. Following the familiarization phase, participants undertook the memory task.

In each trial, seven syllables were presented successively through loudspeakers at a rate of one item per 1,000 ms. Immediately after each list, the recall direction appeared on the computer screen. For the forward-recall group, the instruction «Normal» [Forward] appeared on the screen and participants were required to recall the syllables in their presentation order. For the backward-recall group, the instruction "Inversé » [Backward] appeared on the screen and participants were to recall the syllables in reverse order. Participants were instructed to say «Passe » [Pass] for every syllable they could not recall. Once recall was completed, participants pressed the space bar to initiate the next trial. The experimental session lasted approximately $30 \mathrm{~min}$.

\section{Results}

Responses were scored using a strict recall criterion: A syllable was scored as correct if it was correctly pronounced and recalled in its proper serial position. For all analyses, each repeated list was yoked to the following nonrepeated list so that equal numbers of repeated and nonrepeated trials were included in the analyses. In all analyses, a .05 level of significance was adopted and the Greenhouse-Geisser correction was applied when the sphericity criterion was not met. We analyzed recall performance as a function of serial position, the gradient of improvement over repetitions and participants' response times.

Serial positions Recall performance as a function of serial position is presented in Fig. 1. Figure 1 shows typical serialposition curves, with the forward-recall group showing a larger primacy effect and a weaker recency effect than the backward-recall group. For both groups, recall performance was higher for the repeated than for the nonrepeated lists. A mixed analysis of variance (ANOVA) with two within-subject factors, Repetition (two levels; repeated, nonrepeated) and Serial Position (seven levels; 1 to 7), and one betweensubjects factor, Recall Direction (two levels; forward, backward), was performed on the proportions of correct recall. The analysis confirmed that repeated lists were recalled better than nonrepeated lists, $F(1,38)=100.61, p<.001, \eta_{\mathrm{p}}{ }^{2}=.73$, and that recall performance varied as a function of serial position, $F(6,228)=46.81, p<.001, \eta_{\mathrm{p}}{ }^{2}=.55$. Recall performance did not vary as a function of recall direction, $F<1$. The interaction between repetition and serial position was significant, $F(6$, $228)=2.61, p=.036, \eta_{\mathrm{p}}{ }^{2}=.06$. The interaction between recall direction and serial position was also significant, $F(6,228)=$ $67.54, p<.001, \eta_{\mathrm{p}}{ }^{2}=.64$, suggesting that the forward-recall group yielded a greater primacy effect but a weaker recency effect than the backward-recall group. The analysis revealed a significant interaction between repetition and recall direction, $F(1,38)=10.55, p=.002, \eta_{\mathrm{p}}{ }^{2}=.22$, reflecting a larger difference between repeated and nonrepeated lists in the forwardthan backward-recall group. Paired-sample $t$ tests showed higher recall performance for repeated than for nonrepeated lists for both the forward-recall group, $t(19)=9.27, p<.001, d$

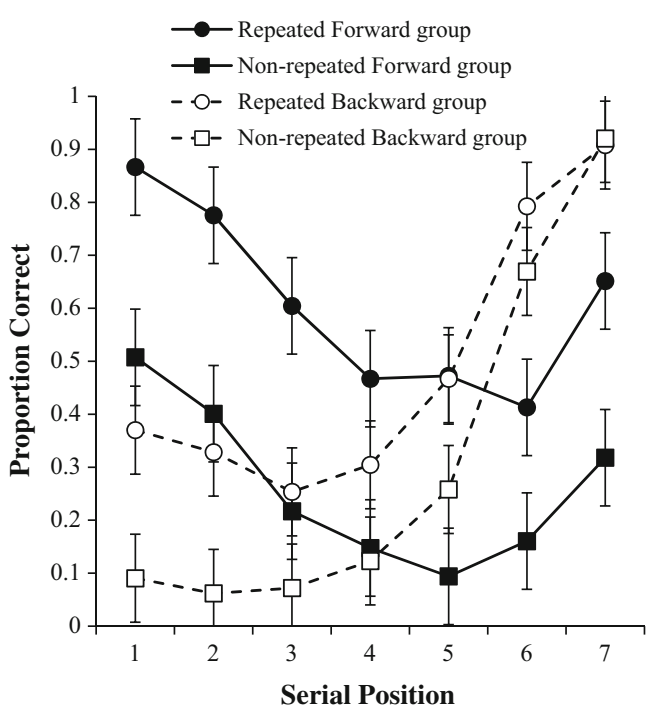

Fig. 1 Proportions of correct recall as a function of serial position for the repeated and nonrepeated lists in the forward- and backward-recall groups of Experiment 1. Error bars represent $95 \%$ confidence intervals calculated separately for the forward- and backward-recall groups (Masson \& Loftus, 2003) 
$=1.07$, and the backward-recall group, $t(19)=4.86, p<.001$, $d=0.51$. The three-way interaction between repetition, recall direction, and serial position was not significant, $F(6,228)=$ $1.27, p=.273, \eta_{\mathrm{p}}{ }^{2}=.03$.

Gradients of improvement For each trial, we computed the mean proportions of correct recall. The 16 repeated trials and their yoked nonrepeated trials were divided into eight blocks. Recall performance for the repeated and nonrepeated trials as a function block is displayed in the top panel of Fig. 2, where each marker represents the mean proportion of correct recall for two repeated trials or their yoked nonrepeated trials. We computed linear regressions on the mean proportion of correct recall in each block, averaged across participants (see Fig. 2, top panel). The regressions, computed separately for the repeated and nonrepeated trials in each recall group, confirmed that recall performance increased as a function of block for the repeated, but not for the nonrepeated, trials. Performance for the repeated trials increased significantly, by .061 per block for the forward-recall group and by .053 per block for the
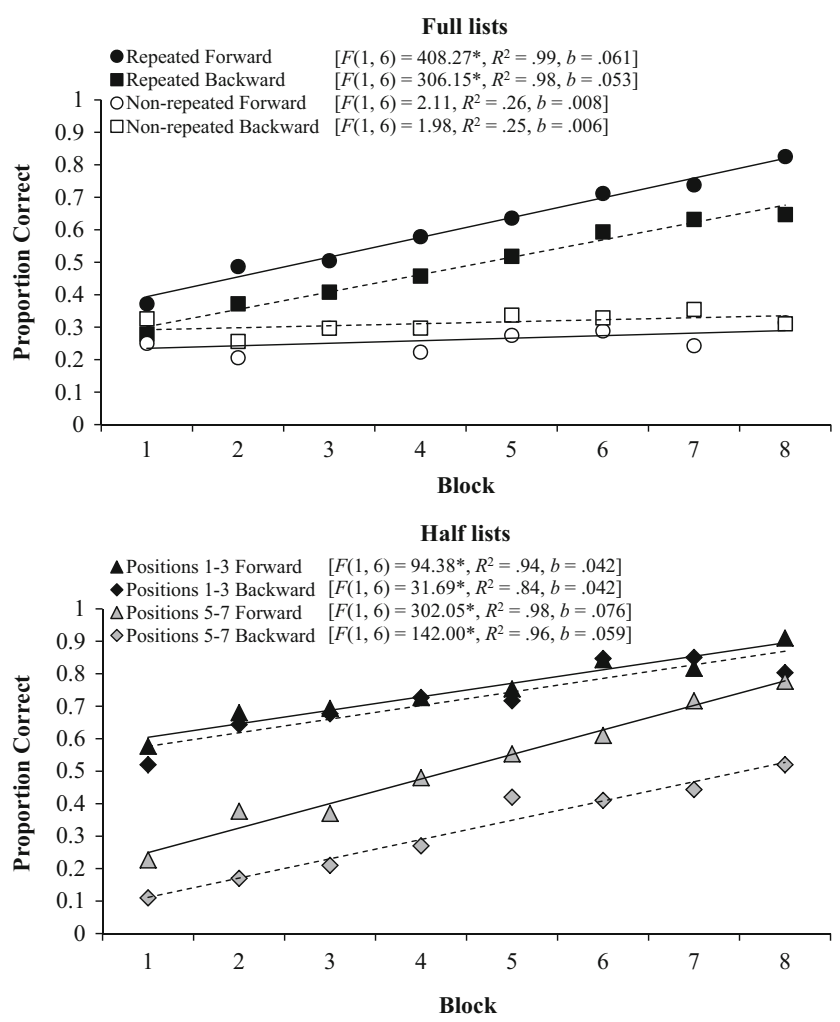

Fig. 2 Proportions of correct recall as a function of block. The top panel shows the full repeated and nonrepeated lists in the forward- and backward-recall groups of Experiment 1. The bottom panel shows the half lists at Serial Positions 1-3 or 5-7 in the forward- and backwardrecall groups of Experiment 1. Linear regressions were computed separately for the repeated and nonrepeated lists and for Serial Positions 1-3 and 5-7 in the forward-recall (solid lines) and backward-recall (dashed lines) groups. Asterisks indicate significant gradients of improvement $(p<.001)$ backward-recall group. The gradients of improvement were used as the dependent variable in a mixed-design ANOVA with Repetition (two levels; repeated, nonrepeated) as a within-subjects factor and Recall Direction (two levels; forward, backward) as a between-subjects factor. The analysis revealed a significant main effect of repetition, $F(1,38)=$ $67.18, p<.001, \eta_{\mathrm{p}}{ }^{2}=.64$, with the repeated lists showing a greater rate of improvement than the nonrepeated lists. The main effect of recall direction was not significant, $F<1$, nor was the interaction between repetition and recall direction, $F<$ 1.

Despite similar gradients of improvement for repeated lists in forward and backward recall, it can be argued that those are due to different learning processes. Across blocks, learning could occur cumulatively from one endpoint of the list to the other endpoint, and this learning process would change as a function of recall direction. According to this view, in forward recall, participants would learn the first serial positions faster than the last ones, whereas the opposite situation would occur in backward recall. This possibility was tested by computing the gradients of improvement on list halves - that is, for the early Serial Positions 1-3 and the final Serial Positions 5-7. Recall performance for list halves as a function of block is presented in the bottom panel of Fig. 2. The linear regressions, computed separately for the list halves in each recall group, confirmed that recall performance increased as a function of block for all lists. For instance, performance for Serial Positions 1-3 significantly increased by .042 per block in both the forward- and backward-recall groups. For Positions 5-7, recall performance significantly increased by .076 for the forward-recall group, whereas it increased by .059 for the backward-recall group. A mixed-design ANOVA with List Half (two levels; Serial Positions 1 to 3, 5 to 7) as a withinsubjects factor and Recall Direction (two levels; forward, backward) as a between-subjects factor was performed on the gradients of improvement. The analysis revealed that Serial Positions 5-7 showed higher gradients of improvement than did Serial Positions $1-3, F(1,38)=6.92, p=.012, \eta_{\mathrm{p}}{ }^{2}=$ .15 . The main effect of recall direction, as well as the interaction between recall direction and list half, was not significant, all $F_{\mathrm{S}}<1$.

Response times Response times more than three standard deviations from the participants' mean were removed from the analysis (11.76\%). As can be seen in Fig. 1, the proportions of correct responses at the early serial positions for backward lists and the middle serial positions for forward lists are so low for nonrepeated lists that response time estimates would be unreliable. To increase the number of observations, we collapsed together all correct and incorrect responses. Such a procedure gives more reliable estimates of response times, since otherwise most of the data would emanate from learned Hebb lists. Moreover, the analysis was restricted to Output 
Positions 3 to 7 , since it has been suggested that the first output items during backward recall could be recalled straight from memory, since they were heard most recently (see Anders \& Lillyquist, 1971; St Clair-Thompson \& Allen, 2013). As is illustrated in Fig. 1, this idea is supported by the very high proportions of correct recall for the last two serial-position items in backward recall. Response times as a function of output order are presented in Fig. 3. A mixed ANOVA with Repetition (two levels; repeated, nonrepeated) and Output Position (five levels; 3 to 7 ) as within-subjects factors and Recall Direction (two levels; forward, backward) as a between-subjects factor revealed that response times varied significantly as a function of output position, $F(2.612$, $91.410)=16.91, p<.001, \eta_{\mathrm{p}}{ }^{2}=.33$, and as a function of recall direction, $F(1,35)=4.91, p=.033, \eta_{\mathrm{p}}{ }^{2}=.12$. The main effect of repetition was not significant, $F<1$. The interaction between output position and recall direction was significant, $F(4$, $140)=2.45, p=.05, \eta_{\mathrm{p}}{ }^{2}=.07$. Neither the interaction between repetition and recall direction, $F(1,35)=1.90, p=.177, \eta_{\mathrm{p}}{ }^{2}=$ .05 , nor the interaction between repetition and output position, $F<1$, was significant. The triple interaction between repetition, output position, and recall direction was not significant, $F(4,140)=1.46, p=.217, \eta_{\mathrm{p}}{ }^{2}=.04$.

\section{Discussion}

Experiment 1 showed typical serial-position curves, with oral presentation yielding a stronger recency but a weaker primacy effect in backward than in forward recall (see Hulme et al., 1997; Li \& Lewandowsky, 1995; Madigan, 1971). Our analysis of response times showed a significant difference between forward and backward recall. Thomas et al. (2003) reported that response times decreased as a function of serial position in backward recall, suggesting that backward recall consisted of a series of forward scans before the recall of every

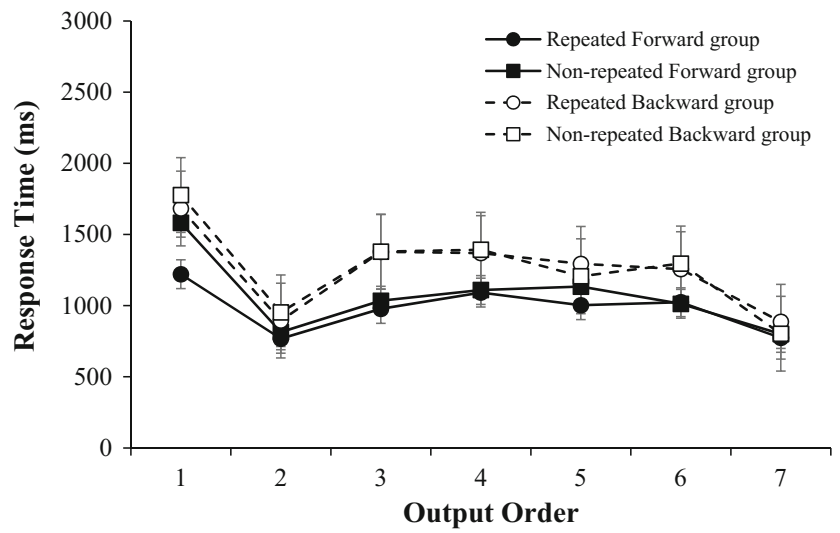

Fig. 3 Response times as a function of output order for the repeated and nonrepeated lists in the forward- and backward-recall groups of Experiment 1. Error bars represent $95 \%$ confidence intervals calculated separately for the forward- and backward-recall groups (Masson \& Loftus, 2003) single item. A visual inspection of Fig. 3, however, suggests that contrarily to Thomas et al., response times in backward recall tended to increase and decrease as a function of output positions. This pattern of output time is more in line with the hypothesis that in backward recall, participants perform a series of covert forward recalls before they output groups of items. This hypothesis is more fully presented in the General Discussion.

Importantly, a Hebb repetition effect was observed irrespective of recall direction. The slopes for both recall directions showed similar learning rates, suggesting that long-term learning occurred irrespective of recall direction. One possibility is that participants learned one input (perceived) list and one output (recalled) list. The forward-recall group would only have had to learn one list, as the input and output list were the same. Participants in the backward recall group would have learned two different lists - that is, one forwardgoing input list and one backward-going output list. As was shown by Couture, Lafond, and Tremblay (2008; Lafond, Tremblay, \& Parmentier, 2010), participants are indeed able to learn output sequences that are different from the perceived sequence.

\section{Experiment 2}

In Experiment 1, a Hebb repetition effect like that typically observed in forward recall was observed in backward recall, even though the production order differed from the order in which the sequence was presented. This suggests that the overt production order per se is not critical for producing a Hebb repetition effect. It might also suggest that participants learn different input and output sequences. In Experiment 2, we investigated whether changing the recall direction of the repeated lists between trials would interfere with learning. If the role of overt language production is critical for long-term learning, changing overt production orders across repetitions should affect the learning rate of the repeated list. If participants learn different input and output lists, it should also be reflected in the learning rate of the repeated lists, since fewer recall opportunities in each recall direction would be offered to participants.

Moreover, by varying recall direction between trials, the impact of foreknowledge could be assessed. Using the word length effect, a working memory benchmark effect, Surprenant et al. (2011) tested whether foreknowledge of the recall direction influenced results. Surprenant et al. contrasted conditions in which the recall direction was known before list presentation, either by using a block presentation similar to that in our first experiment or by indicating recall direction before each list presentation, with a condition in which the recall direction was known only after list presentation. The same pattern of results was found in both cases. Experiment 
2 would allow us to test whether this pattern of results extends to a situation involving long-term sequence learning.

\section{Method}

Participants Twenty French-speaking students (11 men, nine women; mean age $=21.2, S D=1.9$ ) from Université de Moncton participated in the experiment in exchange for class credit. All participants were naive to the purpose of the study; no participants had taken part in Experiment 1.

Materials and procedure The materials and procedure used in Experiment 2 were the same as those in Experiment 1, except that the recall direction varied quasirandomly between trials. Half of the trials required a forward recall, whereas the other half required a backward recall. Within one block, which consists of two consecutive repeated lists and their yoked nonrepeated list, one repeated list was randomly assigned to the forward-recall condition and the other was assigned to the backward-recall condition. Between the nonrepeated lists immediately preceding and following each repeated list, one was randomly assigned to one of the two recall conditions, and the remaining nonrepeated list was assigned to the other recall condition. This was done to ensure that each repeated list would be yoked to a nonrepeated list in the same recall direction within the same block. The first and last experimental trials both involved nonrepeated lists, one of which was assigned to the forward-recall condition and the other to the backward-recall condition. The recall direction appeared on the computer screen after the presentation of the list.

\section{Results}

Each repeated list was yoked with the nonrepeated list presented either before or after that was to be recalled in the same recall direction. We first analyzed recall performance as a function of serial position, learning rates, and response times. To examine whether the learning rate was affected when recall direction varied between trials, we compared the gradients of improvement with those observed in Experiment 1.

Serial positions Recall performance as a function of serial position is displayed in Fig. 4. The repeated lists show higher recall performance than the nonrepeated lists in both forward and backward recall. A 2 (Repetition: repeated, nonrepeated) $\times 2$ (Recall Direction: forward, backward) $\times 7$ (Serial Position: 1 to 7) repeated measure ANOVA was carried out on the proportions of correct recall. The analysis revealed that the repeated lists were recalled better than the nonrepeated lists, $F(1,19)$ $=36.03, p<.001, \eta_{\mathrm{p}}{ }^{2}=.66$, and that recall performance varied significantly as a function of serial position, $F(6,114)=22.25$, $p<.001, \eta_{\mathrm{p}}{ }^{2}=.54$. The main effect of recall direction was not significant, $F<1$. As expected, the interaction between recall

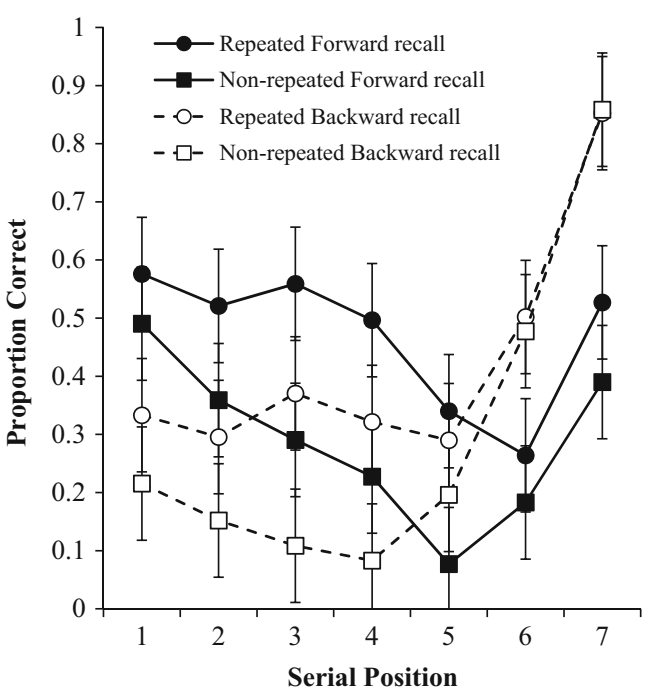

Fig. 4 Proportions of correct recall as a function of serial position for the repeated and nonrepeated lists in the forward- and backward-recall trials of Experiment 2. Error bars represent 95\% confidence intervals (Masson \& Loftus, 2003)

direction and serial position was significant, $F(6,114)=$ 49.08, $p<.001, \eta_{\mathrm{p}}{ }^{2}=.72$. Neither the interaction between repetition and serial position, $F(6,114)=2.37, p=.060, \eta_{\mathrm{p}}{ }^{2}$ $=.11$, nor the critical interaction between recall direction and repetition, $F(1,19)=2.75, p=.114, \eta_{\mathrm{p}}{ }^{2}=.13$, was significant. The three-way interaction between repetition, recall direction, and serial position was not significant, $F<1$.

Gradients of improvement The 16 repeated trials and their associated nonrepeated trials were divided into eight blocks. Each block included one forward repeated trial and one backward repeated trial. Recall performance for the repeated and nonrepeated trials as a function of block is presented in the top panel of Fig. 5. Recall performance for the repeated lists increased across repetitions, regardless of recall direction. Linear regressions showed significant learning gradients, with recall performance increasing by .051 per block for the forward-recall trials and by .048 per block for the backwardrecall trials. A 2 (Repetition: repeated, nonrepeated) $\times 2$ (Recall Direction: forward, backward) repeated measures ANOVA performed on the gradients of improvement revealed a significant main effect of repetition, $F(1,19)=21.95, p<$ $.001, \eta_{\mathrm{p}}{ }^{2}=.54$, with the repeated lists showing a greater rate of improvement than the nonrepeated lists. The main effect of recall direction, as well as the interaction between repetition and recall direction, was not significant, all $F_{\mathrm{S}}<1$.

As in Experiment 1, we computed separate gradients of improvement for the list halves. Recall performance for each list half as a function of block is presented in the bottom panel of Fig. 5. Linear regressions showed similar and significant learning gradients for all types of lists. For the forwardrecalled trials, recall performance increased by .049 per block 

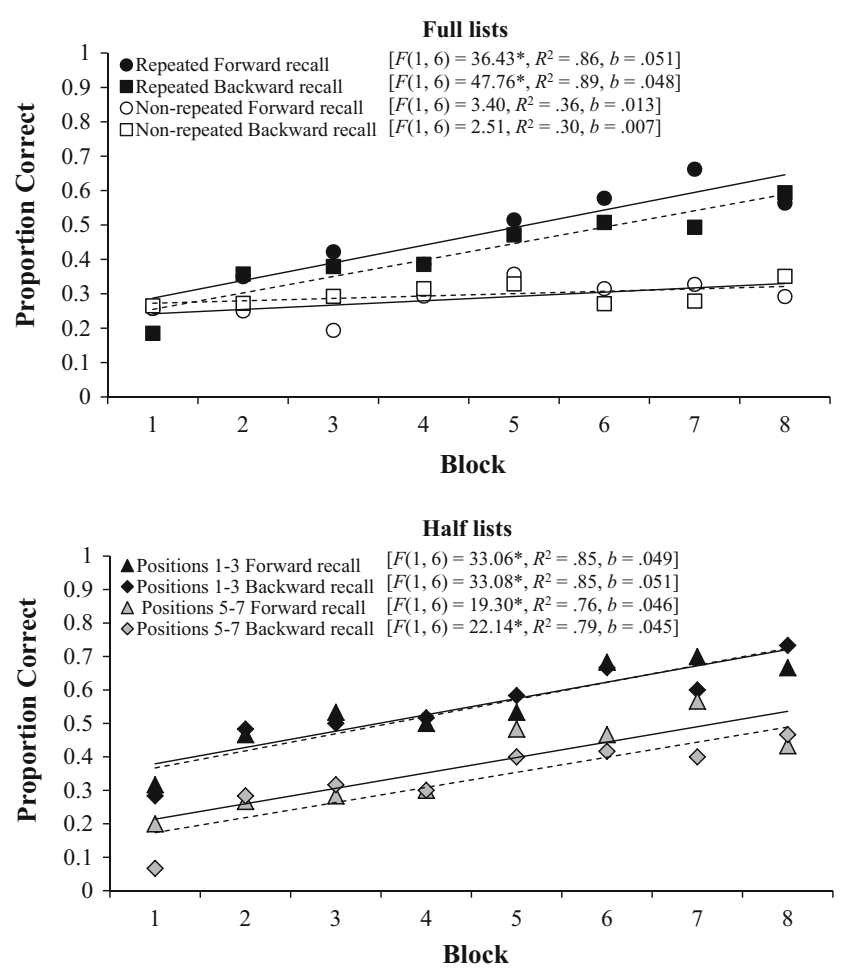

Fig. 5 Proportions of correct recall as a function of block. The top panel shows the full repeated and nonrepeated lists in the forward- and backward-recalled trials of Experiment 2. The bottom panel shows the half lists at Serial Positions 1-3 or 5-7 in the forward- and backwardrecalled trials of Experiment 2. The linear regressions were computed separately for the repeated and nonrepeated trials and for Serial Positions 1-3 and 5-7 in forward recall (solid lines) and backward recall (dashed lines). Asterisks indicate significant gradients of improvement $(p<.05)$

for early serial positions and by .046 per block for final serial positions. Similarly, for the backward-recalled trials, recall performance increased by .051 per block for early serial positions and by .045 per block for final serial positions. A 2 (List Half: Serial Positions 1 to 3,5 to 7 ) $\times 2$ (Recall Direction: forward, backward) repeated measures ANOVA was conducted on the gradients of improvement. The results showed that the gradients of improvement were similar for early and final serial positions, and for forward- and backward-recalled trials (all $F \mathrm{~S}<1$ ). The interaction between list halves and recall direction was also not significant, $F<1$.

Response times Observations more than three standard deviations from the participants' means were removed from the analysis $(8.40 \%)$. As in Experiment 1, all responses (correct and incorrect) were pooled together and the analysis was restricted to Output Positions 3-7. Response times as a function of output order are presented in Fig. 6. A 2 (Repetition: repeated, nonrepeated) $\times 2$ (Recall Direction: forward, backward) $\times 5$ (Output Position: 3 to 7 ) repeated measures ANOVA showed that response times were slower for backward than for forward recall, $F(1,17)=27.39, p<.001, \eta_{\mathrm{p}}{ }^{2}=$

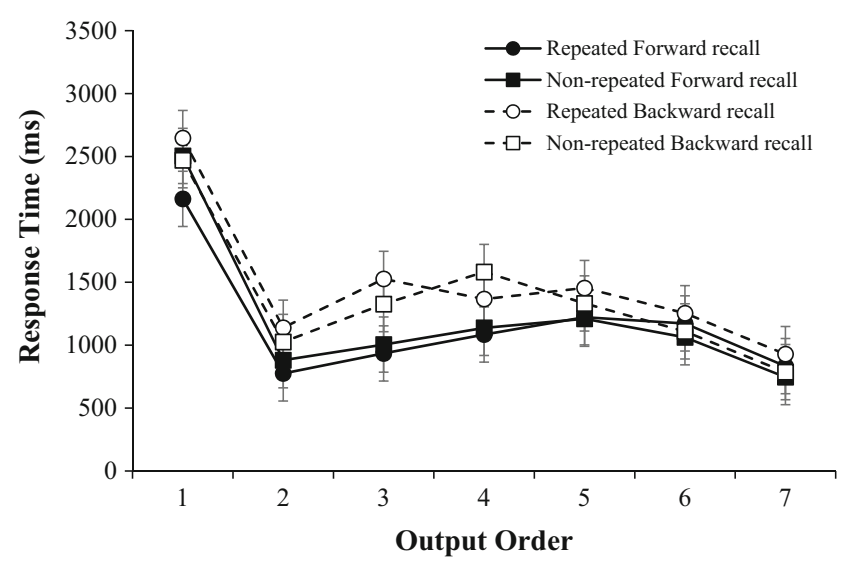

Fig. 6 Response times as a function of output order for the repeated and nonrepeated lists in the forward- and backward-recalled trials of Experiment 2. Error bars represent 95\% confidence intervals (Masson \& Loftus, 2003)

.62 , and varied as a function of output position, $F(2.532$, $43.047)=8.94, p<.001, \eta_{\mathrm{p}}{ }^{2}=.35$. There was no significant difference in terms of response times between repeated and nonrepeated lists, $F(1,17)=1.29, p=.271, \eta_{\mathrm{p}}{ }^{2}=.07$. However, the response times varied significantly between output positions as a function of recall direction, $F(2.479,42.138)$ $=5.02, p=.007, \eta_{\mathrm{p}}{ }^{2}=.23$. The interaction between repetition and output position, $F(4,68)=2.39, p=.059, \eta_{\mathrm{p}}{ }^{2}=.12$, as well as the interaction between repetition and recall direction, $F(1,17)=1.02, p=.326, \eta_{\mathrm{p}}{ }^{2}=.06$, was not significant. The triple interaction between repetition, output position, and recall direction was not significant, $F(2.577,43.809)=1.15, p=$ $.336, \eta_{\mathrm{p}}{ }^{2}=.06$.

\section{Comparison of learning rates between Experiments 1 and}

2 The analysis of the gradients of improvement revealed a nonsignificant main effect of recall direction, suggesting similar learning rates in forward and backward recall. To examine whether the learning rate was affected by randomly varying the recall direction across trials, we compared the gradients of improvement in Experiment 1 to those in Experiment 2. We first compared the gradients of improvement from participants in the forward-recall group in Experiment 1 to the gradients of improvement from the forward-recalled trials in Experiment 2 (see the top panel of Fig. 7). A mixed-design ANOVA with Repetition (two levels: repeated, nonrepeated) as a withinsubjects factor and Group (two levels: Exp. 1, Exp. 2) as a between-subjects factor revealed a significant main effect of repetition, $F(1,38)=44.21, p<.001, \eta_{\mathrm{p}}{ }^{2}=.54$, with the repeated trials showing greater rates of improvement than the nonrepeated trials. The main effect of group, $F<1$, and the interaction between repetition and group, $F(1,38)=1.08$, $p=.306, \eta_{\mathrm{p}}{ }^{2}=.03$, were not significant.

We then compared the gradients of improvement of participants in the backward-recall group in Experiment 1 to those from the backward-recalled trials in Experiment 2 (see the 
bottom panel of Fig. 7). A 2 (Repetition: repeated, nonrepeated) $\times 2$ (Group: Exp. 1, Exp. 2) mixed-design ANOVA showed higher rates of improvement for repeated than for nonrepeated trials, $F(1,38)=44.76, p<.001, \eta_{p}{ }^{2}=.54$. The main effect of group and the interaction between repetition and group were not significant, all $F_{\mathrm{S}}<1$. $^{1}$

\section{Discussion}

As in Experiment 1, backward recall yielded a stronger recency effect and a weaker primacy effect than forward recall. Importantly, Experiment 2 showed the typical Hebb repetition effects in both forward and backward recall. The interexperiment analysis revealed that learning slopes were comparable in Experiments 1 and 2, suggesting that similar learning took place whether or not the recall direction varied between trials. Those results also showed that foreknowledge of recall direction was not mediating the results observed in Experiment 1 (see also Surprenant et al., 2011). It must also be noted that Experiment 2 only provided half of the recall opportunities for the repeated sequence in each recall direction when compared to Experiment 1. Since we obtained similar learning rates in the two experiments, it thus remains unlikely that sequence learning during backward recall relies solely on learning different input and output sequences (see Couture et al., 2008; Lafond et al., 2010).

\section{General discussion}

The objective of the present experiments was to study the role of overt language production in the Hebb repetition effect by using verbal materials with no a-priori associations while manipulating the order in which the lists were output. In Experiment 1, we observed a Hebb repetition effect in backward recall. To our knowledge, we are the first to observe a Hebb repetition effect using a backward-recall procedure. This result indicates that learning is possible even when the output order does not match input order. Moreover, the

\footnotetext{
${ }^{1}$ Because it is not possible to argue for a null effect using null-hypothesis significance testing, we computed a Bayesian repeated measures ANOVA using JASP (Jasp Team, Version 0.8.0.0). For the forward-recall comparison, following recommendations from Rouder, Morey, Speckman, and Province (2012), we found evidence in favor of the alternative hypothesis for the main effect of repetition, and evidence in favor of the null hypothesis for the main effect of group. The main-effects model was also preferred to the interaction model by a Bayes factor of 1.88 . This is evidence against the hypothesis that repetition and group interacted in the learning rates. The same pattern of results was observed in the backward-recall analysis. We found evidence in favor of the alternative hypothesis for the main effect of repetition, and evidence in favor of the null hypothesis for the main effect of group. Again, the main-effect model was preferred to the interaction model by a Bayes factor of 2.59. This stands as evidence against the hypothesis that repetition and group interact in the learning rates.
}
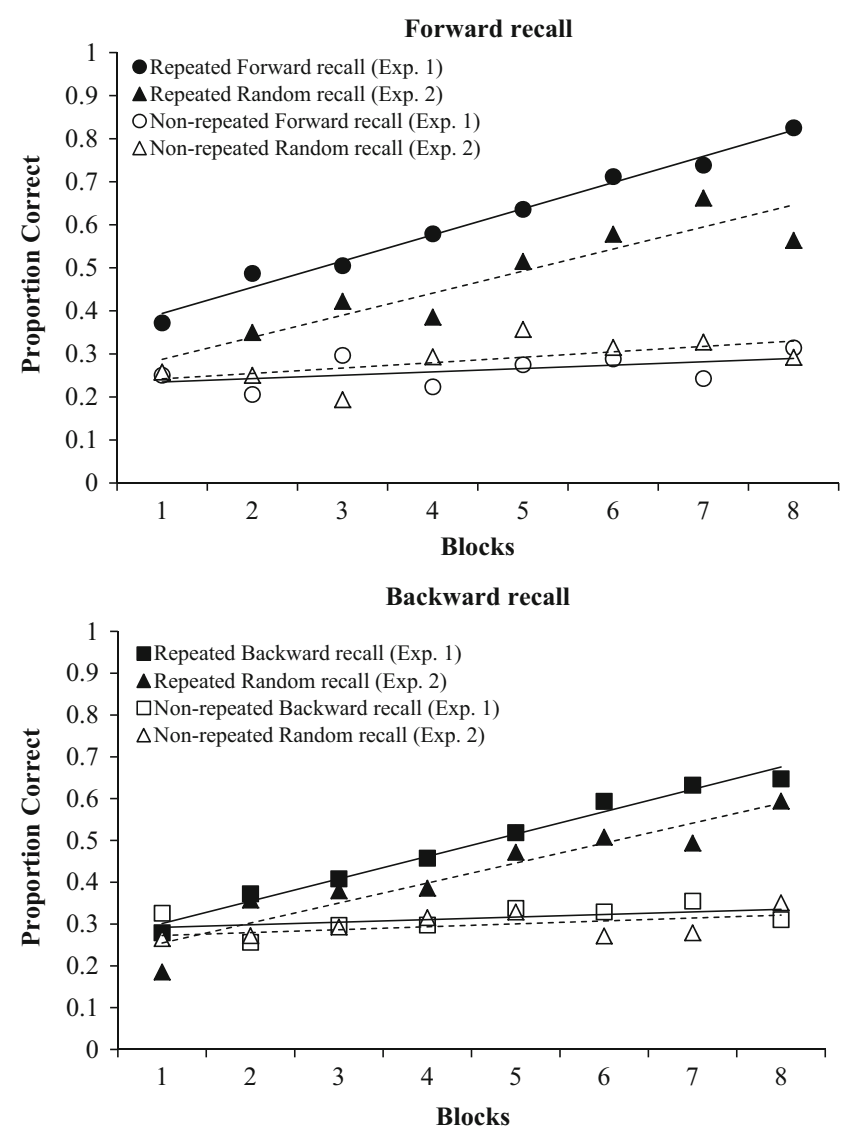

Fig. 7 Proportions of correct recall for the repeated and nonrepeated lists as a function of block in Experiments 1 and 2. The top panel shows the forward-recall group in Experiment 1 and the forward-recalled lists in Experiment 2. The bottom panel shows the backward-recall group in Experiment 1 and the backward-recalled lists in Experiment 2

learning rate in backward recall was similar to that observed in forward recall both at the list level and at the sublevel of list halves. In Experiment 2, we manipulated recall order across repetitions; the analysis showed similar learning between forward and backward recall. A comparison between Experiments 1 and 2 revealed that learning was similar in both experiments, even though in Experiment 2 we provided only half the overt production opportunities for the repeated sequence in each recall direction that had been available in Experiment 1. This suggests that the extent of learning is not influenced by whether overt production order varies across repetitions or not.

The similar learning rates for lists halves in both recall directions rule out an explanation of the Hebb repetition effect based on selective covert language production. For instance, Oberauer and Meyer (2009) speculated that participants may choose to retrieve and rearticulate the subset of list items that are best represented in immediate memory. Because there is a strong primacy effect in forward recall, participants would typically limit their rehearsal to the first presented items (see also Tan \& Ward, 2008). In backward recall, because of the 
large recency effect, participants would instead selectively rehearse the last items. In Experiment 1, this could easily occur because presentation order was blocked, whereas in Experiment 2, this process could only occur during the recall period. Despite its appeal, this hypothesis was not supported by our data. In effect, we found no interaction between recall direction and list half in Experiment 1 or 2.

The similar learning rate observed in both experiments in backward and forward recall further strengthened the view that Hebb learning is a good experimental analogue to wordform learning (e.g., Page \& Norris, 2009). In effect, it has been assumed that backward recall is an executive demanding operation (Szmalec \& Vandierendonck, 2007; Szmalec, Vandierendonck, \& Kemps, 2005). Under this view, the similar learning rate in both recall direction parallel results found with word-form learning showing that it is independent of executive control (Smalle et al., 2016; Szmalec, Page, \& Duyck, 2012).

Importantly, the finding that learning is relatively unaffected by recall direction suggests a limited role of overt language production in the Hebb repetition effect. Indeed, it has been suggested that immediate verbal memory relies on the creation of articulatory sequences (Jones, Hughes, \& Macken, 2006; Jones, Macken, \& Nicholls, 2004). In the Hebb repetition effect, the repeated sequence could have benefited from more practiced coarticulations between items, leading to a faster articulation rate and improved recall performance (see Woodward, Macken, \& Jones, 2008). However, the finding that learning was unaffected in Experiment 2, when the coarticulation order between items changed from trial to trial due to the varying recall direction goes against this idea. Our results are rather in line with studies showing a limited role of language production in the Hebb repetition effect (e.g., Schwartz \& Bryden, 1971). This suggests that the Hebb repetition effect does not rely on the learning of a motor response pattern, and that overt language production is not critical to language learning. This conclusion, however, seems in contradiction with prior studies showing a role of language production in the Hebb repetition effect (Cohen \& Johansson, 1967; Cunningham et al., 1984). For example, Cohen and Johansson showed that when repeated lists were never recalled, learning was equivalent to that observed for nonrepeated lists. However, one possibility is that in their study, it was not the actual overt production of the sequence during recall that was responsible for learning, but that oral recall produced auditory feedback that acted as an additional presentation of the repeated lists.

Our results could be explained using the primacy model (Page \& Norris, 2009). According to the primacy model, the units and their primacy gradients are copied back from the order layer to the occurrence layer, and this feedback would create a chunk over repeated presentations. A sequence is learned when it is associated with a committed chunk.
Because of the primacy gradient, each item can only be retrieved after the retrieval of its predecessor. Therefore, backward recall would occur through a series of covert forward recalls (Page \& Norris, 1998). In line with another study (Bireta et al., 2010), our analyses of response times have ruled out the possibility that participants did series of forward recalls before the output of every single item in the backward-recall condition, as was proposed by Thomas et al. (2003). However, our data is consistent with Page and Norris's (1998) idea of covert forward recalls before the output of groups of items during backward recall. Our response time's pattern (see Figs. 3 and 6) shows quick response times for early output positions, followed by slower response times for middle output positions, and finally quicker response times for the final few output position items. This pattern of response times could be reflecting covert forward recalls and the reversal of items within each group before their overt recall. Under this view, overt production of a sequence is not critical for the emergence of the Hebb effect. Instead, in overt backward recall, covert forward recalls would be sufficient to lead to output learning of the stimulus list. In sum, the primacy model (Page \& Norris, 1998) predicts Hebb learning in backward recall, and its explanation of how backward recall is achieved is consistent with our data.

Another model that has been extended to account for the Hebb repetition effect is Burgess and Hitch's (2006) implementation of the phonological loop. According to this positional model, the presentation of an item activates its associated unit in the input phoneme layer. The activation in the input phoneme layer is then forwarded to the item layer, in which competition for activation between units takes place. Only the most active unit is selected and its activation is forwarded to the output phoneme layer for recall. This model also contains a context signals layer, which provides a unique set of context signals for each sequence. This set of context signals contains information regarding the position of each item within the sequence. During recall, the set of context signals is reinstated, which allows the retrieval of its associated items. Learning would occur through the strengthening of the associations between the items and their context across repetitions.

Burgess and Hitch (2006) have not incorporated backward recall in their model. Regardless, whether the sequence is recalled in forward or backward order, the same context set is activated since the presentation of the sequence remains the same. During backward recall, one possibility is that the context could be reinstated from the end of the sequence (see, e.g., Henson, 1998). The positional codes could therefore be reinstated either in forward or in backward order, depending on recall direction. Thus, backward recall could lead to long-term learning during a Hebb repetition paradigm, because the strengthening of long-term connections during presentation 
would occur independently of recall order. However, our analysis of response times invalidates the use of such strategy, since we observed timing differences between forward and backward recalls in Experiments 1 and 2 (see also Anders \& Lillyquist, 1971; Bireta et al., 2010). Besides, some studies have suggested that the Hebb repetition effect is not caused by strengthening of the context-item associations and that positional models are not suited to explain the long-term learning observed during a Hebb repetition paradigm (Cumming et al., 2003; Hitch, Fastame, \& Flude, 2005; Page et al., 2013).

The processes underlying backward recall are still unclear. Some studies suggested a trade-off between item and order memory at the time of retrieval: The order dimension would receive more attention in backward than in forward recall, and therefore less attention would be given to the item dimension (Bireta et al., 2010; see also Surprenant et al., 2011). Other authors such as St Clair-Thompson and Allen (2013; see also Li \& Lewandowsky, 1995) suggested that backward recall was accomplished through the use of a visuospatial strategy. As per this idea, backward recall would rely on a visuospatial code. During backward recall, visuospatial processes would be used to create a visual image of the sequence. Participants could then retrieve the items in backward order by scanning the visual image starting with the last item presented. St Clair-Thompson and Allen also suggested that phonological memory could be used to recall the first output items (as they were heard most recently). This claim is supported by Anders and Lillyquist's (1971) study, in which participants reported having used such strategy during a backward serial-recall task. St Clair-Thompson and Allen further suggested that as recall progresses, phonological memory would become unreliable, and then visuospatial processes would be used to recall the remaining items in the sequence. Both the item and order trade-off hypothesis and the visuospatial strategy hypothesis suggest that participants employ the same encoding processes, but different recall strategies during forward and backward recall. This is in line with the idea that Hebb learning would occur during the encoding of the repeated sequence and is independent of recall direction.

In sum, our results suggest that the Hebb repetition effect can be observed with stimuli without a-priori associations and is independent of recall direction. The latter finding implies that the overt production of a sequence is not critical for the emergence of a Hebb repetition effect. This is consistent with other studies suggesting that perceptual processes might play a more important role than production processes during longterm learning (Bower \& Winzenz, 1969; Burgess \& Hitch, 2006; Hitch et al., 2009; O'Shea \& Clegg, 2006; Page \& Norris, 2009; Schwartz \& Bryden, 1971). Finally, our results further strengthen the view that Hebbian learning mimics natural word-form learning (Page \& Norris, 2009; Smalle et al., 2016; Szmalec et al., 2009; Szmalec et al., 2012).
Author note This research was supported by a Natural Sciences and Engineering Research Council of Canada graduate scholarship to MarieClaude Guerrette and by discovery grants from the Natural Sciences and Engineering Research Council of Canada to Katherine Guérard and to Jean Saint-Aubin.

\section{References}

Anders, T. R., \& Lillyquist, T. D. (1971). Retrieval time in forward and backward recall. Psychonomic Science, 22, 205-206. doi:10.3758/ bf03332570

Bireta, T. J., Fry, S. E., Jalbert, A., Neath, I., Surprenant, A. M., Tehan, G., \& Tolan, G. A. (2010). Backward recall and benchmark effects of working memory. Memory \& Cognition, 38, 279-291. doi:10.3758/ MC.38.3.279

Bower, G. H., \& Winzenz, D. (1969). Group structure, coding, and memory for digit series. Journal of Experimental Psychology, 80, 1-17. doi: $10.1037 / \mathrm{h} 0027249$

Burgess, N., \& Hitch, G. J. (2006). A revised model of short-term memory and long-term learning of verbal sequences. Journal of Memory and Language, 55, 627-652. doi:10.1016/j.jml.2006.08.005

Cohen, R. L., \& Johansson, B. S. (1967). The activity trace in immediate memory: A re evaluation. Journal of Verbal Learning and Verbal Behavior, 6, 139-143. doi:10.1016/s0022-5371(67)80064-1

Couture, M., Lafond, D., \& Tremblay, S. (2008). Learning correct responses and errors in the Hebb repetition effect: Two faces of the same coin. Journal of Experimental Psychology: Learning, Memory, and Cognition, 34, 524-532. doi:10.1037/0278-7393.34. 3.524

Cumming, N., Page, M., \& Norris, D. (2003). Testing a positional model of the Hebb effect. Memory, 11, 43-63. doi:10.1080/741938175

Cunningham, T. F., Healy, A. F., \& Williams, D. M. (1984). Effects of repetition on short-term retention of order information. Journal of Experimental Psychology: Learning, Memory, and Cognition, 10, 575-597. doi:10.1037/0278-7393.10.4.575

Gathercole, S. E., Service, E., Hitch, G. J., Adams, A.-M., \& Martin, A. J. (1999). Phonological short-term memory and vocabulary development: Further evidence on the nature of the relationship. Applied Cognitive Psychology, 13, 65-77. doi:10.1002/(SICI)10990720(199902)13:1<65::AID-ACP548>3.0.CO;2-O

Hebb, D. O. (1961). Distinctive features of learning in the higher animal. In J. F. Delafresnaye (Ed.), Brain mechanisms and learning: A symposium (pp. 37-51). Oxford: Blackwell Scientific.

Henson, R. N. A. (1998). Short-term memory for serial order: The startend model. Cognitive Psychology, 36, 73-137. doi:10.1006/cogp. 1998.0685

Hitch, G. J., Fastame, M. C., \& Flude, B. (2005). How is the serial order of a verbal sequence coded? Some comparisons between models. Memory, 13, 247-258. doi:10.1080/09658210344000314

Hitch, G. J., Flude, B., \& Burgess, N. (2009). Slave to the rhythm: Experimental tests of a model for verbal short-term memory and long-term sequence learning. Journal of Memory and Language, 61, 97-111. doi:10.1016/j.jml.2009.02.004

Hulme, C., Roodenrys, S., Brown, G. D. A., Schweickert, R., Martin, S., \& Stuart, G. (1997). Word-frequency effects on short-term memory tasks: Evidence for a redintegration process in immediate serial recall. Journal of Experimental Psychology: Learning, Memory, and Cognition, 23, 1217-1232. doi:10.1037/0278-7393.23.5.1217

Jones, D. M., Hughes, R. W., \& Macken, W. J. (2006). Perceptual organization masquerading as phonological storage: Further support for a perceptual-gestural view of short-term memory. Journal of Memory and Language, 54, 265-281. doi:10.1016/j.jml.2005.10. 006 
Jones, D. M., Macken, W. J., \& Nicholls, A. P. (2004). The phonological store of working memory: Is it phonological and is it a store? Journal of Experimental Psychology: Learning, Memory, and Cognition, 30, 656-674. doi:10.1037/0278-7393.30.3.656

Kalm, K., \& Norris, D. (2016). Recall is not necessary for verbal sequence learning. Memory \& Cognition, 44, 104-113. doi:10.3758/ s13421-015-0544-0

Lafond, D., Tremblay, S., \& Parmentier, F. (2010). The ubiquitous nature of the Hebb repetition effect: Error learning mistaken for the absence of sequence learning. Journal of Experimental Psychology: Learning, Memory, and Cognition, 36, 515-522. doi:10.1037/ a0018469

Li, S.-C., \& Lewandowsky, S. (1995). Forward and backward recall: Different retrieval processes. Journal of Experimental Psychology: Learning, Memory, and Cognition, 21, 837-847. doi:10.1037/02787393.21.4.837

Madigan, S. A. (1971). Modality and recall order interactions in shortterm memory for serial order. Journal of Experimental Psychology, 87, 294-296. doi:10.1037/h0030549

Masson, M. J., \& Loftus, G. R. (2003). Using confidence intervals for graphically based data interpretation. Canadian Journal of Experimental Psychology, 57, 203-220. doi:10.1037/h0087426

Mechanic, A. (1964). The responses involved in the rote learning of verbal materials. Journal of Verbal Learning and Verbal Behavior, 3, 30-36. doi:10.1016/s0022-5371(64)80056-6

Mosse, E. K., \& Jarrold, C. (2008). Hebb learning, verbal short-term memory, and the acquisition of phonological forms in children. Quarterly Journal of Experimental Psychology, 61, 505-514. doi: 10.1080/17470210701680779

O'Shea, G., \& Clegg, B. A. (2006). Stimulus and response chunking in the Hebb digits task. Psychological Research, 70, 180-192. doi:10. 1007/s00426-004-0198-1

Oberauer, K., \& Meyer, N. (2009). The contributions of encoding, retention, and recall to the Hebb effect. Memory, 17, 774-781. doi:10. 1080/09658210903107861

Page, M. P. A., Cumming, N., Norris, D., Hitch, G. J., \& McNeil, A. M. (2006). Repetition learning in the immediate serial recall of visual and auditory materials. Journal of Experimental Psychology: Learning, Memory, and Cognition, 32, 716-733. doi:10.1037/ 0278-7393.32.4.716

Page, M. P. A., Cumming, N., Norris, D., McNeil, A. M., \& Hitch, G. J. (2013). Repetition spacing and item-overlap effects in the Hebb repetition task. Journal of Memory and Language, 69, 506-526. doi:10.1016/j.jml.2013.07.001

Page, M. P. A., \& Norris, D. (1998). The primacy model: A new model of immediate serial recall. Psychological Review, 105, 761-781. doi: 10.1037/0033-295x.105.4.761-781

Page, M. P. A., \& Norris, D. (2009). A model linking immediate serial recall, the Hebb repetition effect and the learning of phonological word forms. Philosophical Transactions of the Royal Society B, 364, 3737-3753. doi:10.1098/rstb.2009.0173
Rouder, J. N., Morey, R. D., Speckman, P. L., \& Province, J. M. (2012). Default Bayes factors for ANOVA designs. Journal of Mathematical Psychology, 56, 356-374. doi:10.1016/j.jmp.2012. 08.001

Saint-Aubin, J., Guérard, K., Fiset, S., \& Losier, M.-C. (2015). Learning multiple lists at the same time in the Hebb repetition effect. Canadian Journal of Experimental Psychology, 69, 89-94. doi:10. 1037/cep0000030

Sanders, A. F. (1961). Rehearsal and recall in immediate memory. Ergonomics, 4, 25-34. doi:10.1080/00140136108930504

Schwartz, M., \& Bryden, M. P. (1971). Coding factors in the learning of repeated digit sequences. Journal of Experimental Psychology, 87, 331-334. doi:10.1037/h0030552

Smalle, E. H. M., Bogaerts, L., Simonis, M., Duyck, W., Page, M. P. A., Edwards, M. G., \& Szmalec, A. (2016). Can chunk size differences explain developmental changes in lexical learning? Frontiers in Psychology, 6, 1-14. doi:10.3389/fpsyg.2015.01925

St Clair-Thompson, H. L., \& Allen, R. J. (2013). Are forward and backward recall the same? A dual-task study of digit recall. Memory \& Cognition, 41, 519-532. doi:10.3758/s13421012-0277-2

Surprenant, A. M., Brown, M. A., Jalbert, A., Neath, I., Bireta, T. J., \& Tehan, G. (2011). Backward recall and the word length effect. American Journal of Psychology, 124, 75-86. doi:10.5406/ amerjpsyc.124.1.0075

Szmalec, A., Duyck, W., Vandierendonck, A., Mata, A. B., \& Page, M. P. A. (2009). The Hebb repetition effect as a laboratory analogue of novel word learning. Quarterly Journal of Experimental Psychology, 62, 435-443. doi:10.1080/17470210802386375

Szmalec, A., Page, M. P. A., \& Duyck, W. (2012). The development of long-term lexical representations through Hebb repetition learning. Journal of Memory and Language, 67, 342-354. doi:10.1016/j.jml. 2012.07.001

Szmalec, A., \& Vandierendonck, A. (2007). Estimating the executive demands of a one-back choice reaction time task by means of the selective interference paradigm. Quarterly Journal of Experimental Psychology, 60, 1116-1139. doi:10.1080/17470210600896484

Szmalec, A., Vandierendonck, A., \& Kemps, E. (2005). Response selection involves executive control: Evidence from the selective interference paradigm. Memory \& Cognition, 33, 531-541. doi:10.3758/ bf03193069

Tan, L., \& Ward, G. (2008). Rehearsal in immediate serial recall. Psychonomic Bulletin \& Review, 15, 535-542. doi:10.3758/PBR. 15.3.535

Thomas, J. G., Milner, H. R., \& Haberlandt, K. F. (2003). Forward and backward recall: Different response time patterns, same retrieval order. Psychological Science, 14, 169-174. doi:10.1111/14679280.01437

Woodward, A. J., Macken, W. J., \& Jones, D. M. (2008). Linguistic familiarity in short-term memory: A role for (co-)articulatory fluency? Journal of Memory and Language, 58, 48-65. doi:10.1016/j. jml.2007.07.002 